\title{
$P-V$ criticality in the extended phase space of black holes in massive gravity
}

\author{
Jianfei $\mathrm{Xu}^{a *}$, Li-Ming $\mathrm{Cao}^{a, b \dagger}$, Ya-Peng $\mathrm{Hu}^{b, c \ddagger}$ \\ ${ }^{a}$ Interdisciplinary Center for Theoretical Study \\ University of Science and Technology of China, \\ Hefei, Anhui 230026, China \\ ${ }^{b}$ State Key Laboratory of Theoretical Physics, Institute of Theoretical Physics, \\ Chinese Academy of Sciences, P.O. Box 2735, Beijing 100190, China and \\ ${ }^{c}$ College of Science, Nanjing University of Aeronautics and Astronautics, Nanjing 210016, China
}

(Dated: July 8, 2015)

\begin{abstract}
We study the $P-V$ criticality and phase transition in the extended phase space of charged anti-de Sitter black holes in canonical ensemble of ghost-free massive gravity, where the cosmological constant is viewed as a dynamical pressure of the black hole system. We give the generalized thermodynamic first law and the Smarr relation with massive gravity correction. We find that not only when the horizon topology is spherical but also in the Ricci flat or hyperbolic case, there appear the $P-V$ criticality and phase transition up to the combination $k+c_{0}^{2} c_{2} m^{2}$ in the four-dimensional case, where $k$ characterizes the horizon curvature and $c_{2} m^{2}$ is the coefficient of the second term of massive potential associated with the graviton mass. The positivity of such combination indicate the van der Waals-like phase transition. When the spacetime dimension is larger then four, the Maxwell charge there seems unnecessary for the appearance of critical behavior, but a infinite repulsion effect needed, which can also be realized through negative valued $c_{3} m^{2}$ or $c_{4} m^{2}$, which is third or fourth term of massive potential. When $c_{3} m^{2}$ is positive, a Hawking-Page like black hole to vacuum phase transition is shown in the five-dimensional chargeless case. For the van der Waals like phase transition in four and five spacetime dimensions, we calculate the critical exponents near critical point and find they are the same as those in the van der Waals liquid-gas system.
\end{abstract}

\section{INTRODUCTION}

Over the past decades, the thermodynamics of black holes in asymptotically anti-de Sitter (AdS) space has been widely explored in contrast with its counterpart in asymptotically flat or de Sitter (dS) space since the AdS black holes are thermodynamically stable [1]. Another interesting reason for the focus on AdS black holes is the advent of AdS/CFT correspondence [2-4], which relates a gravity theory in the bulk of an asymptotical AdS space to a conformal field theory without gravity on the boundary of AdS space. According to this holographic dictionary, a bulk AdS black hole corresponds to a boundary finite temperature conformal field theory. At this stage, we can understand a strongly coupled field theory by studying a weakly coupled classical gravity theory. For example, AdS black holes can undergo a so-called Hawking-Page phase transition to a thermal AdS space under a certain Hawking temperature [1]. This phenomenon in gravity theory can be explained as the confinement or deconfinement phase transition in large- $N$ gauge theory [5]. More interesting phenomena show up when we consider the charged AdS black holes $[6,7]$. There exists a first-order phase transition between large black holes and small black holes when the charge is below a critical value. Such phase transitions and critical behaviors of classically charged black holes in AdS space are very similar to a van der Waals liquid-gas phase transition.

Although the thermodynamics of charged black holes in AdS space is similar to the van der Waals system, it should be noted that the corresponding critical behaviors appear in the $Q-\Phi$ diagram, where $Q$ is the charge of the black hole and $\Phi$ is the chemical potential conjugate to the charge [8]. No pressure $P$ or volume $V$ are defined in such a black hole system, while we use the $P-V$ diagram to characterize the van der Waals liquid-gas phase transition. Thus, the analogy is problematic since the charge $Q$ is an extensive quantity and $\Phi$ is an intensive one in the black hole thermodynamics, while $P$ is an intensive quantity and $V$ is an extensive one in the van der Waals system. The way to solve this problem is by including the variation of the cosmological constant $\Lambda$ in the first law of black hole thermodynamics [9-15]. Since the dimension of the cosmological constant over the Newtonian constant $\Lambda / G_{N}$ is equal to the dimension of pressure, it is natural to identify the cosmological constant as the thermodynamical pressure of the system $\left(G_{N}=\hbar=c=k=1\right)$,

$$
P=-\frac{1}{8 \pi} \Lambda=\frac{n(n+1)}{16 \pi l^{2}},
$$

in $n+2$-dimensional spacetime. There are some physical reasons for this identification [16]. First, one can imagine that there exist "more fundamental" theories, where the physical constant, such as the Yukawa coupling, gauge coupling constants, Newtonian constant, or the cosmological constant arise as vacuum expectation values and, hance, can vary. Second, the Smarr

\footnotetext{
* e-mail address: jfxu06@mail.ustc.edu.cn

$\dagger$ e-mail address: caolm@ustc.edu.cn

$\ddagger$ e-mail address: huyp@nuaa.edu.cn
} 
relation obtained by the scaling method becomes inconstant with the first law of black hole thermodynamics unless the variation of $\Lambda$ is included [10]. In addition, once one views the cosmological constant as the thermodynamical pressure, the black hole mass $M$ should be understood as enthalpy rather than the internal energy of the system [10]. Keeping this in mind, we can use the standard thermodynamic identity to get "thermodynamic volume" which is conjugate to the pressure of black holes [16-18]. In this way, people can investigate the $P-V$ critical behaviors of AdS black holes and find exactly the same behaviors as in the van der Waals liquid-gas system. The $P-V$ criticality study of AdS black holes has now been pushed even further to other gravities such as higher-derivative gravities [19-22].

In the above discussion, the gravity theories are either Einstein's general relativity (GR) which has been widely accepted as a correct theory of gravity at low energies or the other possible covariant gravity theories. The most important principle of GR is that it is a theory of a nontrivially interacting massless helicity-2 particle-massless graviton. However, GR is not UV complete. It must be a effective field theory valid at energy up to a cutoff at a certain energy scale beyond which high-energy effects will take place and the gravity theory should be modified [23]. Massive gravity, where the graviton is endowed with mass, is one of the most straightforward modifications of GR. The construction of the linear theory of massive gravity was first given by Fierz and Pauli in 1939 [24]. While at the nonlinear level, the traditional constructions of massive gravity are plagued by the Boulware-Deser (BD) ghost instability [25, 26], up to now, much progress has been made in overcoming the ghost instability. The ghost-free massive gravity was also proposed recently [27, 28]. More recently, a nontrivial black hole solution was found in ghost-free massive gravity with a negative cosmological constant [29]. These black holes' corresponding thermodynamical properties and phase structure have been studied by [30]. The aim of this paper is to analyze the extended phase structure and investigate the $P-V$ critical behavior of charged AdS black holes in canonical ensemble in that ghost-free massive gravity.

This paper is organized as follows. In Sec.II, we present the thermodynamics of massive gravity black holes. We extend the phase space by viewing the cosmological constant as the thermodynamical pressure of the black hole system. In Sec.III, we study the $P-V$ criticality in four-dimensional case. By properly defined thermodynamical quantities, a van der Waals-like phase transition is found and the corresponding critical exponents are calculated. In Sec.IV, We explore the five-dimensional case, where we find that the coefficients of massive potential can also play the role of a Maxwell charge. A Hawking-Page-like phase transition is presented. In Sec.V, we try to understand the physical origin of such van der Waals-like phase transitions in a massive black hole system. The last section is devoted to some conclusions and discussion.

\section{THERMODYNAMICS OF BLACK HOLES IN MASSIVE GRAVITY}

We are considering the following action for an $(n+2)$-dimensional massive gravity [30],

$$
S=\frac{1}{16 \pi} \int d^{n+2} x \sqrt{-g}\left[R+\frac{n(n+1)}{l^{2}}-\frac{1}{4} F^{2}+m^{2} \sum_{i=1}^{4} c_{i} \mathcal{U}_{i}(g, f)\right],
$$

where the last four terms are the massive potential associate with graviton mass, $c_{i}$ are the constants, $f$ is a fixed symmetric tensor called the reference metric, and $\mathcal{U}_{i}$ are symmetric polynomials of the eigenvalue of the $(n+2) \times(n+2)$ matrix $\mathcal{K}_{\nu}^{\mu} \equiv \sqrt{g^{\mu \alpha} f_{\alpha \nu}}$ :

$$
\begin{aligned}
& \mathcal{U}_{1}=[\mathcal{K}] \\
& \mathcal{U}_{2}=[\mathcal{K}]^{2}-\left[\mathcal{K}^{2}\right] \\
& \mathcal{U}_{3}=[\mathcal{K}]^{3}-3[\mathcal{K}]\left[\mathcal{K}^{2}\right]+2\left[\mathcal{K}^{3}\right] \\
& \mathcal{U}_{4}=[\mathcal{K}]^{4}-6\left[\mathcal{K}^{2}\right][\mathcal{K}]^{2}+8\left[\mathcal{K}^{3}\right][\mathcal{K}]+3\left[\mathcal{K}^{2}\right]^{2}-6\left[\mathcal{K}^{4}\right]
\end{aligned}
$$

The square root in $\mathcal{K}$ is understood as the matrix square root, i.e., $(\sqrt{A})_{\nu}^{\mu}(\sqrt{A})_{\lambda}^{\nu}=A_{\nu}^{\mu}$, and the rectangular brackets denote traces- $[\mathcal{K}]=\mathcal{K}_{\mu}^{\mu}$.

The action admits a static black hole solution with the spacetime metric and reference metric as

$$
\begin{gathered}
d s^{2}=-f(r) d t^{2}+f^{-1}(r) d r^{2}+r^{2} h_{i j} d x^{i} d x^{j}, \\
f_{\mu \nu}=\operatorname{diag}\left(0,0, c_{0}^{2} h_{i j}\right),
\end{gathered}
$$

where $c_{0}$ is a positive constant, and $h_{i j} d x^{i} d x^{j}$ is the line element for an Einstein space with constant curvature $n(n-1) k$. Without loss of generality, one may take $k=1,0$, or -1 , corresponding to a spherical, Ricci flat, or hyperbolic topology of the black hole horizon, respectively. According to the reference metric (2.4), we have

$$
\begin{aligned}
& \mathcal{U}_{1}=n c_{0} / r, \\
& \mathcal{U}_{2}=n(n-1) c_{0}^{2} / r^{2}, \\
& \mathcal{U}_{3}=n(n-1)(n-2) c_{0}^{3} / r^{3}, \\
& \mathcal{U}_{4}=n(n-1)(n-2)(n-3) c_{0}^{4} / r^{4} .
\end{aligned}
$$


The metric function $f(r)$ is given by [30]

$$
\begin{aligned}
f(r)= & k+\frac{16 \pi P}{(n+1) n} r^{2}-\frac{16 \pi M}{n V_{n} r^{n-1}}+\frac{(16 \pi Q)^{2}}{2 n(n-1) V_{n}^{2} r^{2(n-1)}}+\frac{c_{0} c_{1} m^{2}}{n} r+c_{0}^{2} c_{2} m^{2} \\
& +\frac{(n-1) c_{0}^{3} c_{3} m^{2}}{r}+\frac{(n-1)(n-2) c_{0}^{4} c_{4} m^{2}}{r^{2}},
\end{aligned}
$$

where $V_{n}$ is the volume of space spanned by coordinates $x^{i}, M$ is the black hole mass, $Q$ is related to the charge of the black hole, and $P=\frac{n(n+1)}{16 \pi l^{2}}$ is the pressure. The black hole horizon is determined by $\left.f(r)\right|_{r=r_{h}}=0$. Thus, the mass $M$ can be expressed in terms of $r_{h}$ as

$$
\begin{aligned}
M= & \frac{n V_{n} r_{h}^{n-1}}{16 \pi}\left[k+\frac{16 \pi P}{(n+1) n} r_{h}^{2}+\frac{(16 \pi Q)^{2}}{2 n(n-1) V_{n}^{2} r_{h}^{2(n-1)}}+\frac{c_{0} c_{1} m^{2}}{n} r_{h}+c_{0}^{2} c_{2} m^{2}\right. \\
& \left.+\frac{(n-1) c_{0}^{3} c_{3} m^{2}}{r_{h}}+\frac{(n-1)(n-2) c_{0}^{4} c_{4} m^{2}}{r_{h}^{2}}\right] .
\end{aligned}
$$

The Hawking temperature of the black hole can be easily obtained by requiring the absence of conical singularity at the horizon in the Euclidean sector of the black hole solution, which is given by

$$
\begin{aligned}
T=\frac{1}{4 \pi} f^{\prime}\left(r_{h}\right)= & \frac{1}{4 \pi r_{h}}\left[(n-1) k+\frac{16 \pi P}{n} r_{h}^{2}-\frac{(16 \pi Q)^{2}}{2 n V_{n}^{2} r_{h}^{2(n-1)}}+c_{0} c_{1} m^{2} r_{h}+(n-1) c_{0}^{2} c_{2} m^{2}\right. \\
& \left.+\frac{(n-1)(n-2) c_{0}^{3} c_{3} m^{2}}{r_{h}}+\frac{(n-1)(n-2)(n-3) c_{0}^{4} c_{4} m^{2}}{r_{h}^{2}}\right] .
\end{aligned}
$$

We are now going to discuss the thermodynamics of black holes in massive gravity in extended phase space by introducing the pressure $P=\frac{n(n+1)}{16 \pi l^{2}}$; the black hole mass $M$ should be viewed as the enthalpy $H \equiv M$ rather than the internal energy of the gravitational system [10]. The other thermodynamic quantities can be obtained through thermodynamic identities. The entropy $S$, thermodynamic volume $V$, and electric potential $\Phi$ are given by

$$
\begin{gathered}
S=\int_{0}^{r_{h}} T^{-1}\left(\frac{\partial H}{\partial r}\right)_{Q, P} d r=\frac{V_{n}}{4} r_{h}^{n}, \\
V=\left(\frac{\partial H}{\partial P}\right)_{S, Q}=\frac{V_{n}}{n+1} r_{h}^{n+1}, \\
\Phi=\left(\frac{\partial H}{\partial Q}\right)_{S, P}=\frac{16 \pi}{(n-1) V_{n} r_{h}^{n-1}} Q .
\end{gathered}
$$

It is easy to check that those thermodynamic quantities obey the following differential equation,

$\mathrm{d} H=T \mathrm{~d} S+V \mathrm{~d} P+\Phi \mathrm{d} Q+\frac{V_{n} c_{0} m^{2} r_{h}^{n}}{16 \pi} \mathrm{d} c_{1}+\frac{n V_{n} c_{0}^{2} m^{2} r_{h}^{n-1}}{16 \pi} \mathrm{d} c_{2}+\frac{n(n-1) V_{n} c_{0}^{3} m^{2} r_{h}^{n-2}}{16 \pi} \mathrm{d} c_{3}+\frac{n(n-1)(n-2) V_{n} c_{0}^{4} m^{2} r_{h}^{n-3}}{16 \pi} \mathrm{d} c_{4}$,

where we have viewed the coupling constants $c_{i}$ as variables. Invoking the scaling method, we can get the Smarr relation for the black hole with $c_{i}$ terms as

$$
(n-1) H=n T S-2 P V+(n-1) \Phi Q-\frac{V_{n} c_{0} c_{1} m^{2}}{16 \pi} r_{h}^{n}+\frac{n(n-1) V_{n} c_{0}^{3} c_{3} m^{2}}{16 \pi} r_{h}^{n-2}+\frac{n(n-1)(n-2) V_{n} c_{0}^{4} c_{4} m^{2}}{8 \pi} r_{h}^{n-3} .
$$

\section{III. $\quad P-V$ CRITICALITY OF FOUR-DIMENSIONAL BLACK HOLES}

In the four-dimensional spacetime case, we can simply set $c_{3}=c_{4}=0$ since we have $\mathcal{U}_{3}=\mathcal{U}_{4}=0$ according to Eq.(2.5) when $n=2$. In this case, the enthalpy (2.7) is reduced to

$$
H=\frac{V_{2} r_{h}}{8 \pi}\left[k+\frac{8 \pi P}{3} r_{h}^{2}+\frac{(8 \pi Q)^{2}}{V_{2}^{2} r_{h}^{2}}+\frac{c_{0} c_{1} m^{2}}{2} r_{h}+c_{0}^{2} c_{2} m^{2}\right] .
$$


And the equation of state of the black holes can be obtained from the Hawking temperature (2.8) in this four-dimensional case as

$$
P=\left(\frac{T}{2}-\frac{c_{0} c_{1} m^{2}}{8 \pi}\right) \frac{1}{r_{h}}-\left(\frac{k}{8 \pi}+\frac{c_{0}^{2} c_{2} m^{2}}{8 \pi}\right) \frac{1}{r_{h}^{2}}+\frac{8 \pi Q^{2}}{V_{2}^{2}} \frac{1}{r_{h}^{4}}
$$

We are now going to study the phase structure of black holes in the canonical ensemble with fixed charge in terms of $P-V$ diagram. Note that the thermodynamic volume $V$ (2.10) is a monotonic function of the horizon radius $r_{h}$, so we can use $r_{h}$ to specify the critical behavior instead of $V$. The critical point is determined as the inflection point in the $P$ - $V$ diagram, i.e.,

$$
\left.\frac{\partial P}{\partial r_{h}}\right|_{r_{h}=r_{h c}, T=T_{c}}=\left.\frac{\partial^{2} P}{\partial r_{h}^{2}}\right|_{r_{h}=r_{h c}, T=T_{c}}=0 .
$$

For further convenience, we would like to denote the coefficients in the equation of state (3.2) as

$$
\begin{aligned}
& w_{1}=\frac{T}{2}-\frac{c_{0} c_{1} m^{2}}{8 \pi} \\
& w_{2}=-\left(\frac{k}{8 \pi}+\frac{c_{0}^{2} c_{2} m^{2}}{8 \pi}\right) \\
& w_{4}=\frac{8 \pi Q^{2}}{V_{2}^{2}} .
\end{aligned}
$$

From now on, we shall denote $w_{1}$ as half of the effective temperature or shifted temperature, for short. Obviously, such a temperature can be negative according to the value of $c_{1} m^{2}$. Thus, the critical point determined by Eq.(3.3) with critical quantities can be calculated as

$$
\begin{gathered}
r_{h c}=\sqrt{-\frac{6 w_{4}}{w_{2}}}, \\
w_{1 c}=-\frac{4}{3} w_{2} \sqrt{-\frac{w_{2}}{6 w_{4}}}, \\
P_{c}=\frac{w_{2}^{2}}{12 w_{4}} .
\end{gathered}
$$

Note that the critical behavior occurs only when $w_{2}<0$. We can easily find a universal relation among critical pressure $P_{c}$, shifted temperature $w_{1 c}$, and horizon radius $r_{h c}$,

$$
\frac{P_{c} r_{h c}}{w_{1 c}}=\frac{3}{8}
$$

This is called the critical coefficient, which keeps the same value as the van der Waals liquid-gas system. However, if we use real the critical temperature $T_{c}$ instead, we cannot get a constant critical coefficient.

The $P-V\left(r_{h}\right)$ diagrams have been drawn in the Fig. 1 with different shifted temperature $w_{1}$. The left plot shows exactly the same behavior as the van der Waals system and has the critical point, while the right plot shows the monotone phase structure according to the different sign of $w_{2}$. Thus, changing the sign of $w_{2}$ can dramatically change the phase diagram. The van der Waals-like phase diagram indicates that there must exist a phase transition when $w_{2}<0$. To clearly specify the phase transition, we shall introduce the Gibbs free energy as a Legendre transformation of enthalpy as

$$
G=H-T S
$$

By using Eq.(2.12), we can immediately get the exterior derivative of Gibbs free energy as $\mathrm{d} G=-S \mathrm{~d} T+V \mathrm{~d} P+\Phi \mathrm{d} Q$, which tell us that the Gibbs free energy will take the minimal value in the equilibrium state when the temperature, pressure, and charge of the system are all held fixed. In Fig. 2, we have plotted the Gibbs free energy as a function of the shifted temperature for various pressures. It can be seen from the left diagram that when $w_{2}<0$ and $P<P_{c}$, there exists a "swallow tail"-type behavior and indicate a first-order phase transition as expected. The "tail" characterizes the unstable state, since the Gibbs-free energy always takes the minimal value for constant temperature, pressure, and electric charge. The "swallow tail" disappears when $P>P_{c}$. The right diagram is monotonically decreasing, and has no conflict with $P-V\left(r_{h}\right)$ statement. There exists no phase transition when $w_{2}>0$. We can see from the $G-w_{1}$ diagrams that the shifted temperature can take a negative value for a stable black hole phase. However, if we plot the $P-V\left(r_{h}\right)$ diagram with negative $w_{1}$, we will find that the stable black hole will have a maximal horizon radius above which the pressure will become negative. 

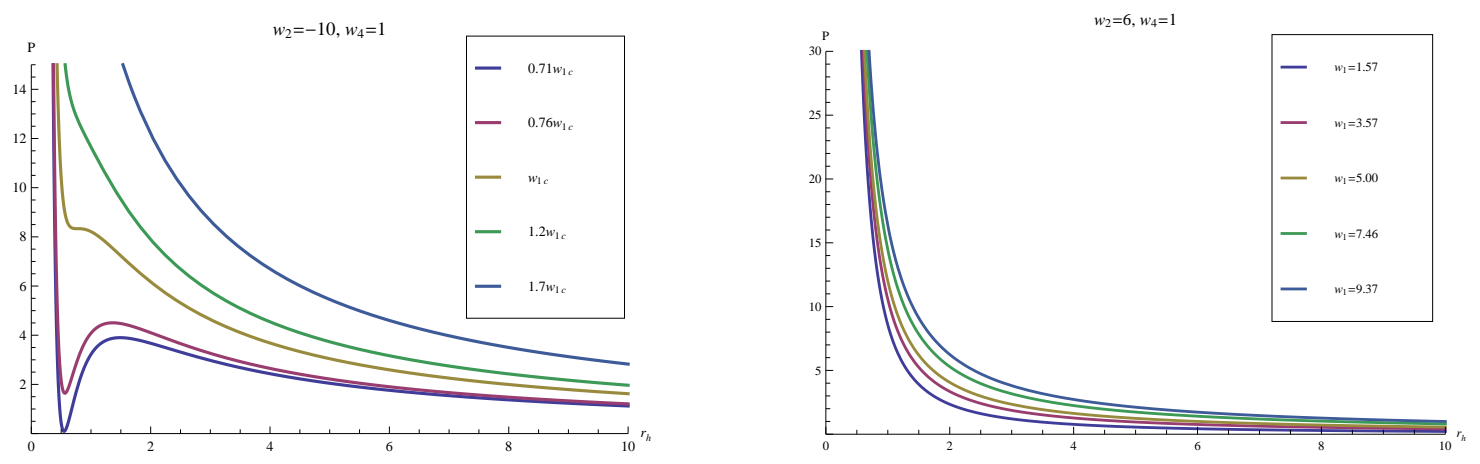

FIG. 1: The four-dimensional $P-V\left(r_{h}\right)$ diagrams for $w_{2}=-10$ and $w_{2}=6$, where we have set $w_{4}=1$ for simplicity. The left diagram shows the van der Waals-like critical behavior, while the right one does not according to the different sign of $w_{2}$.
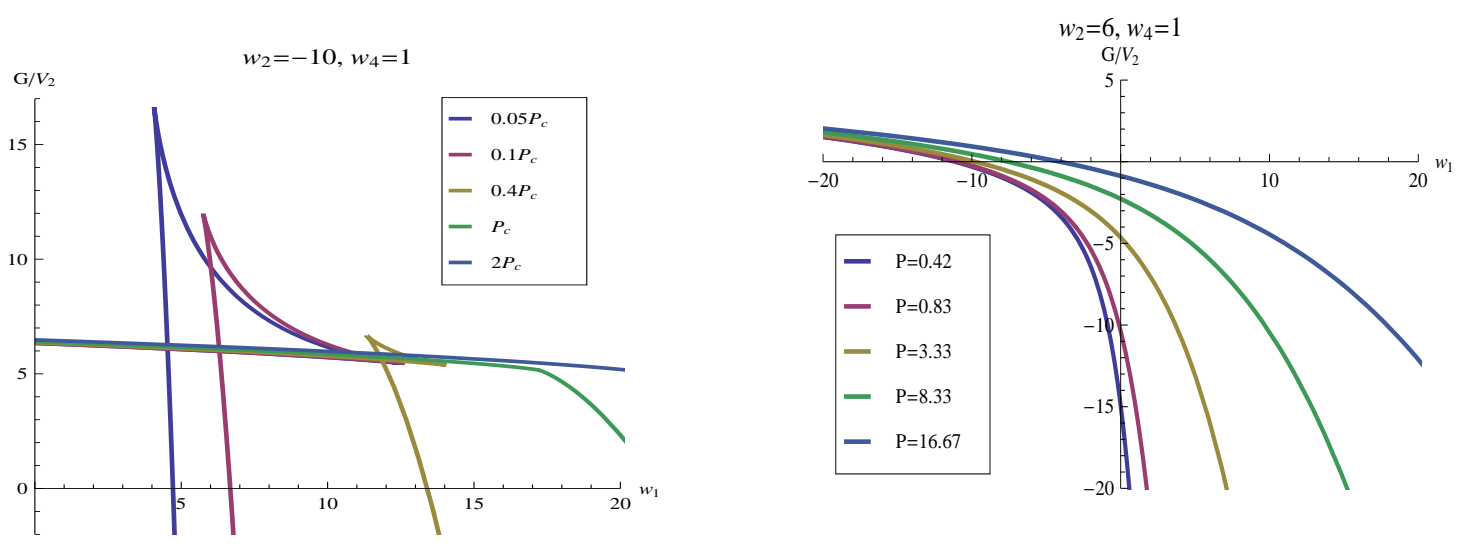

FIG. 2: The four-dimensional Gibbs free energy as a function of shifted temperature for different pressures. The left diagram shows the "swallow tail" behavior while the right one does not according to the different sign of $w_{2}$.

Now let us turn to calculate the critical exponents in the massive gravity black hole system. In the usual thermodynamic system, the critical exponents $\alpha, \beta, \gamma$, and $\delta$ are defined as follows,

$$
\begin{aligned}
C_{v} & \sim\left(-\frac{T-T_{c}}{T_{c}}\right)^{-\alpha}, \\
\frac{v_{g}-v_{l}}{v_{c}} & \sim\left(-\frac{T-T_{c}}{T_{c}}\right)^{\beta}, \\
\kappa_{T} & \sim\left(-\frac{T-T_{c}}{T_{c}}\right)^{-\gamma}, \\
P-P_{c} & \sim\left(v-v_{c}\right)^{\delta},
\end{aligned}
$$

where $C_{v}$ is the isopyknic heat capacity, $v$ stands for specific volume, and $\kappa_{T}$ is isothermal compressibility. The subscript $c$ stands for the critical point.

For our massive gravity black hole system, the size is determined by the horizon radius, and we shall use the following expansion parameters to characterize the critical behavior near the critical point:

$$
\tau=\frac{w_{1}}{w_{1 c}}-1, \quad \epsilon=\frac{r_{h}}{r_{h c}}-1, \quad p^{\prime}=\frac{P}{P_{c}} .
$$

Then we can make the Taylor series expansion near the critical point for the equation of state (3.2) as

$$
p^{\prime}=1+\frac{8}{3} \tau-\frac{8}{3} \tau \epsilon-\frac{4}{3} \epsilon^{3}+\mathcal{O}\left(\tau \epsilon^{2}, \epsilon^{4}\right) .
$$



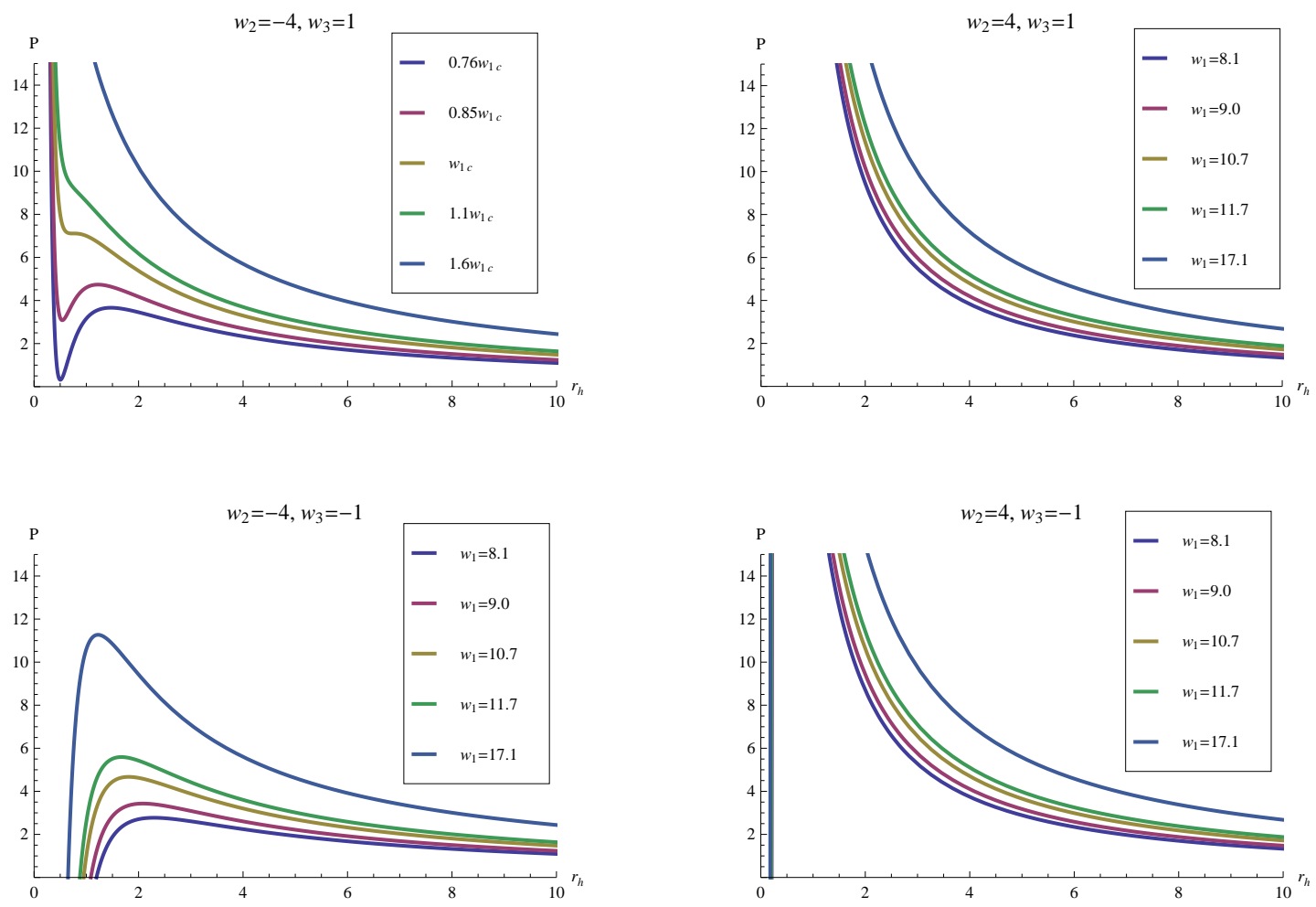

FIG. 3: The five-dimensional $P-V\left(r_{h}\right)$ diagrams with different parametrization. The first diagram shows the van der Waals-like critical behavior when $w_{2}<0$ and $w_{3}>0$.

By using Maxwell's equal area law, we obtain the following equation:

$$
\begin{aligned}
0 & =\int_{\epsilon_{l}}^{\epsilon_{g}}(\epsilon+1)^{3} \frac{\mathrm{d} p^{\prime}}{\mathrm{d} \epsilon} \mathrm{d} \epsilon=\int_{\epsilon_{l}}^{\epsilon_{g}}(\epsilon+1)^{3}\left(-\frac{8}{3} \tau-4 \epsilon^{2}\right) \mathrm{d} \epsilon \\
& =-\frac{8}{3} \tau\left(\epsilon_{g}-\epsilon_{l}\right)-\frac{4}{3}\left(\epsilon_{g}^{3}-\epsilon_{l}^{3}\right)-4 \tau\left(\epsilon_{g}^{2}-\epsilon_{l}^{2}\right)-\frac{8}{3} \tau\left(\epsilon_{g}^{3}-\epsilon_{l}^{3}\right)+\mathcal{O}\left(\epsilon^{4}\right) .
\end{aligned}
$$

On the other hand, the pressures of two phases keep the same value when the phase transition happens:

$$
\left.p^{\prime}\right|_{\epsilon_{l}}=\left.p^{\prime}\right|_{\epsilon_{g}} \Rightarrow-\frac{8}{3} \tau\left(\epsilon_{g}-\epsilon_{l}\right)-\frac{4}{3}\left(\epsilon_{g}^{3}-\epsilon_{l}^{3}\right)+\mathcal{O}\left(\epsilon^{4}\right)=0 .
$$

The above two equations (3.13) and (3.14) have a unique nontrivial solution $\epsilon_{g}>0, \epsilon_{l}<0$ when $\tau<0$. It is easy to find that

$$
\epsilon_{g}-\epsilon_{l}=2 \sqrt{-2 \tau+\mathcal{O}\left(\tau^{2}\right)}
$$

which determines the critical exponent $\beta=1 / 2$. The isothermal compressibility can be calculated as follows

$$
\kappa_{T}=-\left.\left.\frac{1}{(\epsilon+1)^{3}}\left(\frac{\partial(\epsilon+1)^{3}}{\partial P}\right)\right|_{\tau} \propto\left(\frac{\partial p^{\prime}}{\partial \epsilon}\right)^{-1}\right|_{\epsilon=0}=-\frac{3}{8 \tau},
$$

which indicates the critical exponent $\gamma=1$. The difference between the pressure and its critical value near the critical point $\left.\left(p^{\prime}-1\right)\right|_{\tau=0}=-\frac{4}{3} \epsilon^{3}$ tells us that $\delta=3$. The isopyknic heat capacity vanishes since the entropy is also determined by the horizon radius, and then we have $\alpha=0$. The critical exponents satisfy the following thermodynamic scaling laws,

$$
\begin{aligned}
& \alpha+2 \beta+\gamma=2, \quad \alpha+\beta(1+\delta)=2 \\
& \gamma(1+\delta)=(2-\alpha)(\delta-1), \quad \gamma=\beta(\delta-1),
\end{aligned}
$$

which are the same as those in the van der Waals liquid-gas system. 

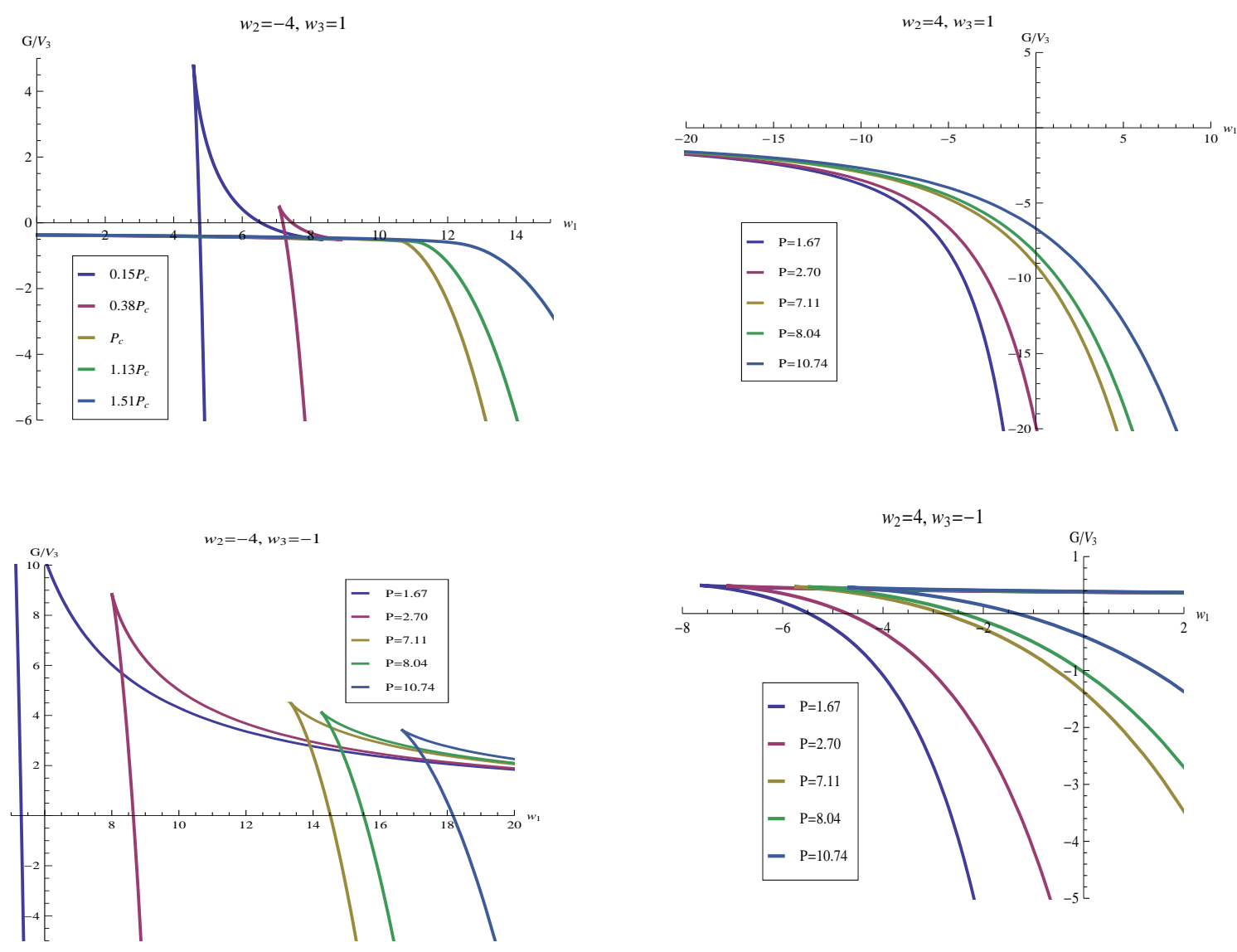

FIG. 4: The five-dimensional Gibbs free energy as a function of shifted temperature with different parametrization. The "swallow tail" behavior appears when $w_{2}<0$ and $w_{3}>0$.

\section{IV. $P$ - $V$ CRITICALITY OF FIVE-DIMENSIONAL NEUTRAL BLACK HOLES}

In the five-dimensional case, we have $n=3$ and $\mathcal{U}_{4}=0$. So we can set $c_{4}=0$ in the metric function. In this section, we will show that the $c_{3} m^{2}$ term in five-dimensional neutral black holes can play a similar role as the charge. We set $Q=0$ for simplicity. The enthalpy (2.7) becomes

$$
H=\frac{3 V_{3} r_{h}^{2}}{16 \pi}\left[k+\frac{4 \pi P}{3} r_{h}^{2}+\frac{c_{0} c_{1} m^{2}}{3} r_{h}+c_{0}^{2} c_{2} m^{2}+\frac{2 c_{0}^{3} c_{3} m^{2}}{r_{h}}\right]
$$

The equation of state is

$$
P=\left(\frac{3}{4} T-\frac{3 c_{0} c_{1} m^{2}}{16 \pi}\right) \frac{1}{r_{h}}-\left(\frac{3 k}{8 \pi}+\frac{3 c_{0}^{2} c_{2} m^{2}}{8 \pi}\right) \frac{1}{r_{h}^{2}}-\frac{3 c_{0}^{3} c_{3} m^{2}}{8 \pi} \frac{1}{r_{h}^{3}}
$$

As in the four-dimensional case, it is convenient to denote

$$
w_{3}=-\frac{c_{0}^{3} c_{3} m^{2}}{8 \pi},
$$

so the equation of state can be put in a neat form according to the definition of $w_{1}$ and $w_{2}$ in Eq.(3.4)

$$
P=\frac{3 w_{1} / 2}{r_{h}}+\frac{3 w_{2}}{r_{h}^{2}}+\frac{3 w_{3}}{r_{h}^{3}} .
$$

The critical point is determined by the vanishing of the first and second derivative of $P$ with respect to $r_{h}$. The critical value of $r_{h}, w_{1}$, and $P$ can be calculated as follows:

$$
r_{h c}=-\frac{3 w_{3}}{w_{2}}
$$



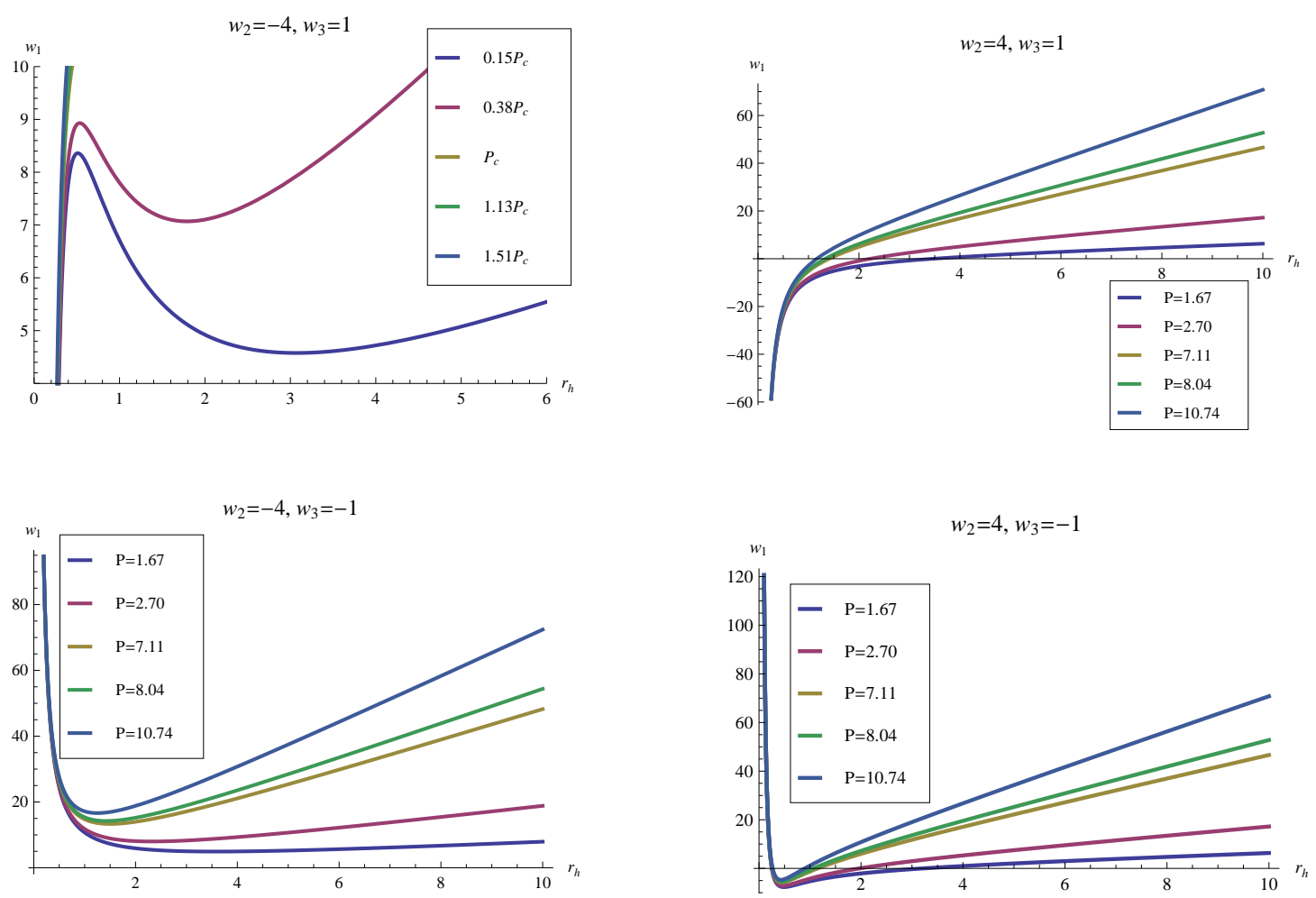

FIG. 5: The 5-dimensional $w_{1}-r_{h}$ diagrams with different parametrization. The negative slop indicate the negative heat capacity and hance the thermodynamic instability.

$$
\begin{gathered}
w_{1 c}=\frac{2 w_{2}^{2}}{3 w_{3}}, \\
P_{c}=-\frac{w_{2}^{3}}{9 w_{3}^{2}} .
\end{gathered}
$$

Similarly, we shall keep $w_{2}<0$ and $w_{3}>0$ when the critical behavior appears, and the critical coefficient in this case is

$$
\frac{P_{c} r_{h c}}{w_{1 c}}=\frac{1}{2} .
$$

By the same token, one can show that the critical exponents in this five-dimensional case are kept the same as those in four dimensions.

One can draw the phase diagram to see the phase structure near the critical point. In the Fig. 3 and 4 , we give the $P$ - $V\left(r_{h}\right)$ and $G-w_{1}$ diagrams according to the different signs of $w_{2}$ and $w_{3}$ in this five-dimensional neutral black hole case.

The positivity of $w_{3}$ in equation of state (4.4) leads to a divergence of pressure when $r_{h} \rightarrow 0$ which is very similar to the charge effect in four dimensions. And the sign of $w_{2}$ determines whether there exists a first-order phase transition or not. For a van der Waals like phase transition, $w_{2}$ must be negatively valued. Interestingly, when $w_{3}$ is negative, in spite of the value of $w_{2}$, there always exists one preiection in the $P-V\left(r_{h}\right)$ diagram, and the pressure drops to zero when the horizon radius decreases to a nonzero value. A more reasonable and physical explanation will be explored in the next section.

In this chargeless case, the vacuum solution should also be taken into account. According to the Hamiltonian approach, the vacuum solution must be the one with zero energy (here is the enthalpy), entropy and, hance, the horizon radius. The Gibbs-free energy of the vacuum solution vanishes by the definition (3.9). The first diagram of the Fig. 4 shows a "swallow tail" behavior when the pressure is below a critical point. Note that only the "tail" may appear in the upper half plane with positive Gibbs-free energy, the thermodynamic stable phase will always be the black hole phase, and the phase space stays the same as the one in four dimensions. This is true in the second diagram as well. In the third and fourth diagram of the Fig. 4 with $w_{3}=-1$, only the part in the lower half plane indicates a stable black hole solution. The shifted temperature has a minimal value below which no black hole solution exists. In fact, when the temperature drops to a certain value larger than the minimal one, the Gibbs-free energy will become larger than zero and a more stable vacuum will take place. This is a Hawking-Page-like phase transition. 
On the other hand, the thermodynamic instability can also be implied by negatively valued heat capacity. The heat capacity of the balck hole system with fixed pressure and charge can be calculated as

$$
\begin{aligned}
C_{P, Q} & =\left(\frac{\partial H}{\partial T}\right)_{P, Q}=T\left(\frac{\partial S}{\partial T}\right)_{P, Q} \\
& =T \frac{\mathrm{d} S}{\mathrm{~d} r_{h}}\left(\frac{\partial T}{\partial r_{h}}\right)_{P, Q}^{-1} .
\end{aligned}
$$

We can see that the sign of $C_{P, Q}$ is determined by the sign of $\left(\frac{\partial T}{\partial r_{h}}\right)_{P, Q}^{-1}$ and, hance, by the sign of $\left(\frac{\partial w_{1}}{\partial r_{h}}\right)_{P, Q}^{-1}$. In the Fig. 5, we plot the the $w_{1}-r_{h}$ diagrams with fixed pressures in this neutral black hole case. We keep the same values of $w_{2}$ and $w_{3}$ as in $G-w_{1}$ diagrams. The negative slope of $w_{1}-r_{h}$ curve indicates a negative heat capacity and, hance, instability. The first diagram of Fig. 5 shows the instability with negative slope when the pressure is below the critical one, which is consistent with the $G$ - $w_{1}$ analysis. The second diagram of Fig. 5 with $w_{2}=4$ and $w_{3}=1$ tells us that there is only one stable black hole phase. The last two diagrams of Fig. 5 are also consistent with the $G$ - $w_{1}$ analysis that there exist unstable black hole phases with $C_{P, Q}<0$.

\section{P- $V$ CRITICALITY OF GENERAL HIGHER-DIMENSIONAL BLACK HOLES}

In this section, we will analyze the $P-V$ criticality of charged black holes in general spacetime dimensions based on the previous analysis of special cases. According to the Hawking temperature, i.e., Eq. (2.8), the equation of state for such charged black holes with general spacetime dimensions is

$$
\begin{aligned}
P= & \left(\frac{n T}{4}-\frac{n c_{0} c_{1} m^{2}}{16 \pi}\right) \frac{1}{r_{h}}-\frac{n(n-1)\left(k+c_{0}^{2} c_{2} m^{2}\right)}{16 \pi} \frac{1}{r_{h}^{2}}-\frac{n(n-1)(n-2) c_{0}^{3} c_{3} m^{2}}{16 \pi} \frac{1}{r_{h}^{3}} \\
& -\frac{n(n-1)(n-2)(n-3) c_{0}^{4} c_{4} m^{2}}{16 \pi} \frac{1}{r_{h}^{4}}+\frac{8 \pi Q^{2}}{V_{n}^{2}} \frac{1}{r_{h}^{2 n}} .
\end{aligned}
$$

Obviously, we can see that the power of $r_{h}$ of the $c_{i}$ terms is independent of spacetime dimensions, while the charge term is not. This ensure that the contributions of every $c_{i}$ term in different spacetime dimensions are qualitatively the same. The things that matter for the diversity of the phase structure or $P-V$ diagram are the value of $c_{i} m^{2}$ s. For example, the $r_{h}$ dependence of the $c_{4} m^{2}$ term is $r_{h}^{-4}$ when $c_{4} m^{2}$ is negatively valued and $n>3$; it gives a chargelike contribution as in four spacetime dimensions. In any spacetime dimension, the charge term plays a dominant role as $r_{h} \rightarrow 0$ since it has the lowest power of $r_{h}$. Note that the charge term has a positive sign, and the pressure will tend to infinity when $r_{h} \rightarrow 0$ as long as the black hole is charged.

Physically, an infinite pressure implies the existence of repulsion interaction. Let us take the van der Waals liquid-gas system as an example. The equation of state is the van der Waals equation

$$
P=\frac{T}{v-b}-\frac{a}{v^{2}}
$$

where $v$ is the so-called specific volume, and $a$ and $b$ are positive constants determined by experiments. In contrast to the ideal gas with $a=b=0$, constants $a$ and $b$ reflect the attraction and repulsion force between molecules, respectively. When $b / v$ is not very large, the van der Waals equation can be written as an Annes equation with series expansion

$$
P=\frac{T}{v}+\frac{b T}{v^{2}}-\frac{a}{v^{2}}+\frac{2 b^{2} T}{v^{3}}+\frac{6 b^{3} T}{v^{4}}+\mathcal{O}\left(v^{-5}\right) .
$$

In the above equation (5.3), the pressure is expressed as a polynomial of $v$, the positive coefficient reflects the repulsion interactions, and the negative coefficient is related to the attraction interactions. As to our state equation of charged black holes in general spacetime dimensions, i.e., Eq. (5.1), if we identify the horizon radius $r_{h}$ as the specific volume, it has a very similar structure as the Annes equation (5.3). The positive sign of the charge term implies a repulsion interaction which could be understood. The value of $c_{i} m^{2} \mathrm{~s}$ cannot be determined by the theory itself. This leaves many possibilities which lead to a plentiful phase structure. For example, if $c_{4} m^{2}$ is negatively valued, then the fourth term in Eq. (5.1) reflects a repulsion interaction, and vice versa. Similar properties can be applied to $c_{3} m^{2}$. The sign of the second term of Eq. (5.1) is determined by $k+c_{0}^{2} c_{2} m^{2}$, which is a joint effect of the horizon topology and $c_{2} m^{2}$. In other words, at least in the thermodynamic sense, the appearance of $c_{2} m^{2}$ mist the contribution of horizon topology. This can also been seen from the metric function (2.6) where the constant term is $k+c_{0}^{2} c_{2} m^{2}$. Thus, unlike with the charged AdS black hole in GR, a hyperbolic or spherical horizon topology does not necessarily imply a repulsion or attraction interaction. It depends on the sign of $k+c_{0}^{2} c_{2} m^{2}$. The appearance of $c_{1} m^{2}$ seems exotic since no such term appears in the usual Annes equation (5.3). At present, we can only understand it as a correction to the Hawking temperature.

Now we can conclude that the appearance of the critical behaviors or the first-order phase transitions is the results of competition of repulsion and attraction interactions between some unknown degree of freedom. For such phase transitions, an infinite repulsion interaction is necessary when the horizon radius tends to zero. 


\section{CONCLUSIONS AND DISCUSSION}

In this paper, we have found that in the context of massive gravity, there also exists the van der Waals-like phase transition in the extended phase space of charged AdS black holes when the cosmological constant is identified as the thermodynamical pressure. For such identification, the black hole mass must be viewed as thermal enthalpy rather than the internal energy of the gravitational system. Then we use the standard thermodynamic identities to obtain the entropy, volume, and electric potential. The entropy keeps the one quarter of the horizon area law as in GR. In contrast with black holes in GR, the phase structure becomes richer due to the appearance of the massive potential associated with the graviton mass. There are four terms in the massive potential. As to the spatial reference metric black holes, the contribution of these four terms in some thermodynamic quantities like enthalpy or temperature is dimensional dependent. The Smarr relation is modified by this massive potential.

In the four-dimensional case, only the first and second terms appear in the thermal enthalpy as well as the equation of state. In the canonical ensemble, the van der Waals-like phase transition happens when $k+c_{0}^{2} c_{2} m^{2}>0$. In this first-order phase transition, we calculate the critical exponents and find that these are the same as in the van der Waals liquid-gas phase transition. It is worth mentioning that we have used the shifted temperature rather than the real one to characterize the critical point. In this sense, we find the same critical coefficient as lin the iquid-gas phase transition. The figure of Gibbs-free energy as a function of shifted temperature for constant pressure shows the "swallow tail" behavior when $k+c_{0}^{2} c_{2} m^{2}>0$. The "tail" characterizes the unstable state with larger values of Gibbs-free energy for constant temperature, pressure, and electric charge.

In the five-dimensional case, the third term in the massive potential contributes. In order to see the effect of the term $c_{3} m^{2}$, we simply set $Q=0$. There also can undergo a van der Waals-like phase transition if, and only if $k+c_{0}^{2} c_{2} m^{2}>0$ and $c_{3} m^{2}<0$. Qualitatively, there are four possible shapes in the $P-V$ diagram according to the different sign of $k+c_{0}^{2} c_{2} m^{2}$ and $c_{3} m^{2}$. When $c_{3} m^{2}>0$, there always exists a Hawking-Page-like phase transition at a certain temperature from the black hole state to the vacuum state.

Generally, we can express the thermodynamical pressure as polynomial in terms of horizon radius. By contrasting with the Annes equation, we find that it is natural to identify the horizon radius as the specific volume. As a polynomial, the positive coefficient reflects the repulsion interactions and the negative coefficient is related to the attraction interactions. The appearance of the critical behaviors or the first-order phase transitions are the result of the competition of repulsion and attraction interactions between some unknown degree of freedom. In particular, the $c_{2} m^{2}$ term will mix the contribution of horizon topology and the $c_{1} m^{2}$ term can be viewed as a correction to the Hawking temperature.

Finally, we would like to mention that the black hole solution in massive gravity is tightly related to the choice of the reference metric. Whether the thermodynamic properties depend on the choice of the reference metric is another interesting topic that deserves future study.

\section{ACKNOWLEDGMENTS}

We would like to thank Professor Rong-Gen Cai for his valuable disscussion and comments. This work was supported in part by the National Natural Science Foundation of China under Grants No. 11205148, No. 11235010, and No. 11105004, the Fundamental Research Funds for the Central Universities under Grant No. NS2015073, and Shanghai Key Laboratory of Particle Physics and Cosmology under Grant No. 11DZ2260700.

[1] S. W. Hawking and D. N. Page, Commun. Math. Phys. 87, 577 (1983).

[2] J. M. Maldacena, Int. J. Theor. Phys. 38, 1113 (1999) [Adv. Theor. Math. Phys. 2, 231 (1998)] [hep-th/9711200].

[3] S. S. Gubser, I. R. Klebanov and A. M. Polyakov, Phys. Lett. B 428, 105 (1998) [hep-th/9802109].

[4] E. Witten, Adv. Theor. Math. Phys. 2, 253 (1998) [hep-th/9802150].

[5] E. Witten, Adv. Theor. Math. Phys. 2, 505 (1998) [hep-th/9803131].

[6] A. Chamblin, R. Emparan, C. V. Johnson and R. C. Myers, Phys. Rev. D 60, 064018 (1999) [hep-th/9902170].

[7] A. Chamblin, R. Emparan, C. V. Johnson and R. C. Myers, Phys. Rev. D 60, 104026 (1999) [hep-th/9904197].

[8] J. y. Shen, R. G. Cai, B. Wang and R. K. Su, Int. J. Mod. Phys. A 22, 11 (2007) [gr-qc/0512035].

[9] M. M. Caldarelli, G. Cognola and D. Klemm, Class. Quant. Grav. 17, 399 (2000) [hep-th/9908022].

[10] D. Kastor, S. Ray and J. Traschen, Class. Quant. Grav. 26, 195011 (2009) [arXiv:0904.2765 [hep-th]].

[11] B. P. Dolan, Class. Quant. Grav. 28, 125020 (2011) [arXiv:1008.5023 [gr-qc]].

[12] B. P. Dolan, Class. Quant. Grav. 28, 235017 (2011) [arXiv:1106.6260 [gr-qc]].

[13] B. P. Dolan, Phys. Rev. D 84, 127503 (2011) [arXiv:1109.0198 [gr-qc]].

[14] M. Cvetic, G. W. Gibbons, D. Kubiznak and C. N. Pope, Phys. Rev. D 84, 024037 (2011) [arXiv:1012.2888 [hep-th]].

[15] H. Lu, Y. Pang, C. N. Pope and J. F. Vazquez-Poritz, Phys. Rev. D 86, 044011 (2012) [arXiv:1204.1062 [hep-th]].

[16] D. Kubiznak and R. B. Mann, JHEP 1207, 033 (2012) [arXiv:1205.0559 [hep-th]].

[17] M. K. Parikh, Phys. Rev. D 73, 124021 (2006) [hep-th/0508108].

[18] W. Ballik and K. Lake, Phys. Rev. D 88, no. 10, 104038 (2013) [arXiv:1310.1935 [gr-qc]].

[19] S. W. Wei and Y. X. Liu, Phys. Rev. D 87, no. 4, 044014 (2013) [arXiv:1209.1707 [gr-qc]]. 
[20] H. Xu, W. Xu and L. Zhao, Eur. Phys. J. C 74, no. 9, 3074 (2014) [arXiv:1405.4143 [gr-qc]].

[21] W. Xu and L. Zhao, Phys. Lett. B 736, 214 (2014) [arXiv:1405.7665 [gr-qc]].

[22] R. G. Cai, L. M. Cao, L. Li and R. Q. Yang, JHEP 1309, 005 (2013) [arXiv:1306.6233 [gr-qc]].

[23] K. Hinterbichler, Rev. Mod. Phys. 84, 671 (2012) [arXiv:1105.3735 [hep-th]].

[24] M. Fierz and W. Pauli, Proc. Roy. Soc. Lond A 173, 211 (1939).

[25] D. G. Boulware and S. Deser, Phys. Lett. B 40, 227 (1972).

[26] D. G. Boulware and S. Deser, Phys. Rev. D 6, 3368 (1972).

[27] S. F. Hassan and R. A. Rosen, Phys. Rev. Lett. 108, 041101 (2012) [arXiv:1106.3344 [hep-th]].

[28] S. F. Hassan, R. A. Rosen and A. Schmidt-May, JHEP 1202, 026 (2012) [arXiv:1109.3230 [hep-th]].

[29] D. Vegh, arXiv:1301.0537 [hep-th].

[30] R. G. Cai, Y. P. Hu, Q. Y. Pan and Y. L. Zhang, Phys. Rev. D 91, 024032 (2015) [arXiv:1409.2369 [hep-th]]. 